\title{
Transport Spectroscopy of Single-Walled Carbon Nanotubes
}

\author{
David H. Cobden, Marc Bockrath, Nasreen Chopra, Alex Zettl and Paul L. McEuen \\ Department of Physics, University of California at Berkeley, and Lawrence Berkeley National Laboratory MS 2-200, Berkeley,
} CA94720

\author{
Andrew Rinzler, Andreas Thess and Richard E. Smalley \\ Center for Nanoscale Science and Technology, Rice Quantum Institute, and Departments of Chemistry and Physics, Mail \\ Stop 100, Rice University, P.O. Box 1892, Houston, TX 77251
}

(June 18, 2021, e-mail: dcobden@physics.berkeley.edu)

\begin{abstract}
We have performed transport spectroscopy on individual ropes of single-walled carbon nanotubes. We find that the levels are Zeeman split in a magnetic field, with a g-factor of $2.04 \pm 0.05$. The observed pattern of peak splittings indicates the parit $\mathrm{y}$ of the number of electrons on the dot. In one device there are also signs of the presence of a second dot. We observe features which resemble anticrossings between quantum levels in the two dots, which may be formed from separate conducting nanotubes within the rope.
\end{abstract}

In recent transport measurements on individual singlewalled carbon nanotubes [1] and bundles, or "ropes", of single-walled carbon nanotubes [2], evidence was found that the electrons were confined to one-dimensional (1D) channels [3] of a finite length. The devices behaved as quantum dots, and it was therefore possible to use nonlinear current-voltage $(I-V)$ measurements to reveal excited states, whose spacing was found to be consistent with expectations for partic les in a 1D box. Also, in a magnetic field Tans et al. [1] observed an excited state which moved relative to the ground state at a rate corresponding to a g-factor of $1.9 \pm 0.2$, consistent with the expected free-electron Zeeman shi ft. A surprising aspect of their data was the apparent absence of the expected splitting of the ground state transition.

Here we present results of detailed transport spectroscopy (measurement of $d I / d V$ as a function of bias $V$ and gate voltage $V_{g}$ ) at millikelvin temperatures on a short rope segment. We find that a magnetic field produces a Zeeman splitting with a g-f actor of $2.04 \pm 0.05$. The pattern of splittings of the peaks in $d I / d V$ allows us to assign even or odd parity to the number of electrons on the dot [4] over a range of more than ten consecutive Coulomb peaks. We also find evidence of the presence of a second weakly conducting dot, and observe apparent anticrossings of the levels in two dots. The two dots may be formed from two coupled conducting nanotubes within the rope.

To make a device [2] we deposit the nanotube material [5] sparsely on $\mathrm{SiO}_{2}$, locate an isolated rope relative to prefabricated alignment marks using an atomic force microscope (AFM), and deposit chrome-gold contacts over it by e lectron-beam lithography. In an improvement on our earlier technique we use a degenerately doped silicon substrate to act as a metallic gate. An image of a device is inset to Figure 1. The diameter of the rope is about 5 $\mathrm{nm}$, so that it consists of arou nd ten nanotubes. Only one segment of this rope, to which source and drain leads are shown schematically attached, conducted.
Figure 1 shows the small-signal conductance, $G=$ $d I /\left.d V\right|_{V=0}$, at $100 \mathrm{mK}$, as function of gate voltage $V_{g}$ applied to the conducting substrate. It exhibits a series of sharp peaks of varying height and spacing. If measurements are limited to a range of about $2 \mathrm{~V}$ in $V_{g}$ these peaks are highly reproducible. The results of applying a dc bias $V$ are best represented in a grayscale plot of $d I / d V$. Figure 2 shows such a plot for the region of $V_{g}$ around the three peaks labeled P, Q and R in Figure 1 . Each dark line in this figure is the locus of a peak in $d I / d V$. Each of the peaks at $V=0$ becomes a cross in the $V-V_{g}$ plane, with extra structure inside it which is different for each cross.

The effect of a magnetic field $B$ parallel to the rope is shown in Figure 3 (a) for the cross formed by peak Q. Most of the lines, such as those marked $\mathrm{X}$ and $\mathrm{Y}$ here, split into pairs ( $\mathrm{X}$ splits into $\mathrm{X}_{1}$ and $\mathrm{X}_{2}$ ) whose separation is proportional to $B$. Some lines however (such as line Z) do not split. In the remainder of the data we observe the following pattern: on successive crosses alternately the leftmost or the rightmost line does not split. The splitting is the only discernible effect of $B$ on the positions of any of the lines.

As discussed previously [1,2], the peaks in $G$ in Figure 1 can be explained by the Coulomb blockade model of a quantum dot [6]. In this model peaks in $d I / d V$ (dark lines in Figure 2) are attributed to the alignment of quantum levels in the dot with the Fermi levels in the contacts. The sketches in Figure 3 (b) illustrate this with regard to the $B=0$ grayscale in Figure 3 (a). Each level is indicated by two horizontal lines to represent the spin degeneracy. The bi as $\mathrm{V}$ is applied to the left contact with the right contact grounded. We take the number of electrons $N$ on the dot to the left of cross Q to be even, for reasons given below. The lowest available level for $N+1$ electrons is doubly degenerate, and lie s a distance $U+\delta E$ above the highest filled level for $N$ electrons, where $U$ is the charging energy and $\delta E$ is the level spacing. Along line $\mathrm{X}$ this level aligns with the Fermi level of 
the source, while along line $\mathrm{Z}$ it aligns with that of the drain. Line $\mathrm{Y}$ is associated with alignment of the source with an excited level, as indicated in Figure 3 (b). The difference in $V_{g}$ between $\mathrm{X}$ and $\mathrm{Z}$ at any bias $V$ produces a change of $V$ in the electrostatic po-tential of the dot. From this w e deduce that a change in $V_{g}$ should be multiplied by $\alpha=0.09$ to obtain the corresponding change in dot potential.

From the typical separation of lines like $\mathrm{Y}$ and $\mathrm{X}$ we estimate the average level spacing to be $\delta E \sim 5 \mathrm{meV}$, while equating the average spacing in $V_{g}$ of the peaks in $G$ with $(U+\delta E) / e \alpha$ we obtain $U \sim 25 \mathrm{meV}$. We may compare these parameters with the expected properties of a quantum dot formed from a single nanotube. Assuming the nanotube acts as a 1D box for the electrons, the average level spacing should be $\delta E=\pi \hbar v_{F} / 2 L=(0.5$ $\mathrm{eV} \mathrm{nm}) / L$, where $v_{F}=4.8 \times 10^{5} \mathrm{~ms}^{-1}$ [] is the Fermi velocity, $L$ is the length and the 2 accounts for the two $1 \mathrm{D}$ bands at the Fermi energy. Also, the charging energy of a small object of size $L$ incorporated into an electronic device is typically $U \sim(1.5 \mathrm{eV} \mathrm{nm}) / L$. For $L=100 \mathrm{~nm}$, these estimates yield $\delta E=5 \mathrm{meV}$ and $U \sim 15 \mathrm{meV}$. We conclude that a nanotube of length approximately equal to the distance between the contacts (in this case lithographically defined as $200 \mathrm{~nm}$ ) should have both a level spacing and a charging energy similar to the measured values. The same was true in our previous rope devices [2], while in the work of Tans et al. [1] the level spacing and charging energy were consistent with $L=3 \mu \mathrm{m}$, the entire length of the nanotube.

Most ropes consist of tubes with a range of chiralities [5.9, and the fraction of metallic tubes may be small 10,11. Hence the origin of the dot in our device is likely to be a single conducting nanotube within the rope, bounded lengthwise by the metal contacts. Barriers at the contacts may be created by contamination or damage during fabrication, or by interaction between the contact metal and the tube 8 .

The effect of magnetic field in Figure 3 (a) can be understood within the Coulomb blockade model as resulting from the Zeeman splitting. The fact that the only effect of $B$ is to split some of the lines is consistent with the absence of orbital coupling to a magnetic field expected for a 1D conductor. Figure 3 (c) indicates the lifting of the spin degeneracy in Figure 3 (b) by the Zeeman energy $g \mu_{B} B$. With $N$ even, the $N+1$ 'th electron must tunnel from the source into the next available orbit al level. For $B>0$ the spin-down state aligns with the source along line $\mathrm{X}_{1}$ and the spin-up state along $\mathrm{X}_{2}$. The separation of $\mathrm{X}_{1}$ and $\mathrm{X}_{2}$ in $V_{g}$ is $g \mu_{B} B / \alpha$. From a series of measurements at different $B$ we obtain $g=2.04 \mathrm{pm} 0.05$, in agreement with the g-factor of $2.001 \pm 0.001$ yielded by electron spin resonance measurements on bulk nanotube material [12].

The line marked $\mathrm{Z}$ on the right of the cross, which does not split, corresponds to electrons tunneling out of the spin-down $N+1$ 'th level to Fermi level in the drain. In this case the spin-up state produces no line, because once the lower-energy spin-dow $\mathrm{n}$ state is below the drain Fermi level it is permanently occupied, excluding occupancy of the spin-up state. Similar arguments show that if $N$ is odd, the opposite pattern is seen: the line on the right of the cross splits while the one on the left does not [4. We find that the pattern of splittings alternates between these two types over at least ten crosses. This implies that the electrons are added sequentially to the dot just as expected in the single particle picture, first spin d own then spin up for each level.

The picture described earlier of a rope as a collection of mostly insulating nanotubes with an occasional metallic one helps us interpret some observed deviations from the single-dot Coulomb blockade model. Figure 4 shows grayscale plots covering peaks G, H, K, L and M in Figure 1. Faint additional lines can be seen in between the crosses, moving at a shallow angle to the $V_{g}$ axis. These extra lines are associated with an indistinct cross (not shown) interstitial between peaks I and J. This other cr oss indicates the presence of a second dot, which we can deduce is poorly coupled to at least one of the contacts. From a closer analysis we can estimate the interdot charging energy to be $\sim 2 \mathrm{meV}$. The second dot has a smaller capacitance to the ga te than the first so its levels move at a different rate with $V_{g}$. If the dots are tunnel-coupled the states in them should hybridize and the resulting levels anticross. We suggest that the anticrossings apparent in Figure 4 are of this nature, and we can estimate a coupling of $\sim 1 \mathrm{meV}$ from the minimum separation at the anticrossing. It is possible that the second dot is a different conducting nanotube within the rope. Since the coupling between adjacent tubes is expected to be large [7], the two tubes may not be in direct contact. Finally we remark that a variety of aspects of the data, some of which are evident on close inspection of Figure 4, remain mysterious and hint at exciting discoveries to come in this novel system.

In conclusion, we have performed detailed transport spectroscopy on an individual rope of single-walled carbon nanotubes. We observe a level spacing and Zeeman splitting consistent with a single-particle model of a finite $1 \mathrm{D}$ conductor, and see possible e vidence for tunnel coupling between different conducting nanotubes within the rope.

\section{ACKNOWLEDGMENTS}

We would like to acknowledge useful discussions with Cees Dekker, Sander Tans and Steven Louie. The work at LBNL was supported by the Office of Naval Research, Order No. N00014-95-F-0099 through the U.S. Department of Energy under Contract No. DE-AC03-76SF00098, and by the Packard Foundation. The work at Rice was 
funded in part by the National Science Foundation and the Robert A. Welch Foundation.

FIG. 1. Small-signal two-terminal conductance $G$ of a single-walled nanotube rope vs gate voltage $V_{g}$. Inset: AFM image indicating the leads used in the measurement.

FIG. 2. Grayscale plot of $d I / d V$ (dark $=$ more positive) vs bias $V$ and $V_{g}$ for peaks $\mathrm{P}, \mathrm{Q}$ and $\mathrm{R}$ in Figure 1 .

FIG. 3. (a) Grayscale plot of $d I / d V$ for peak Q in Figure 2 at a series of magnetic fields. (b) Coulomb-blockade model for the features (dark lines) in (a) at $B=0$. (c) Effect of Zeeman splitting on the features at $B>0$.

FIG. 4. Grayscale plots of $d I / d V$ for peaks $\mathrm{G}, \mathrm{H}, \mathrm{K}, \mathrm{L}$ and $\mathrm{M}$ in Figure 1, showing anticrossings and other distortions possibly related to the presence of a second dot.
[1] Sander S. Tans et al., Nature 386, 474 (1997).

[2] Marc Bockrath et al., Science 275, 1922 (1997).

[3] N. Hamada, S. Sawada, and A. Oshiyama, Phys. Rev. Lett. 68, 1579 (1992); R. Saito et al., Appl. Phys. Lett. 60, 2204 (1992).

[4] D. C. Ralph, C. T. Black, and M. Tinkham, Phys. Rev. Lett. 74, 3241 (1995).

[5] Andreas Thess et al., Science 273, 483 (1996).

[6] Leo P. Kouwenhoven et al., Proc. ASI on Mesoscopic Electron Transport, eds. L. P. Kouwenhoven, G. Schon and L. L. Sohn, (Kluwer 1997) (1997).

[7] Young-Kyun Kwonet al., preprint (1997).

[8] Paul Delaney et al., preprint (1997).

[9] Qin Lu-Chang et al., Chem. Phys. Lett. 268, 101 (1997).

[10] Cees Dekker, private communication.

[11] Charles Lieber, private communication.

[12] J. C. Charlier, X. Gonze, and J. P. Michenaud, Europhys. Lett. 29, 43 (1995). 


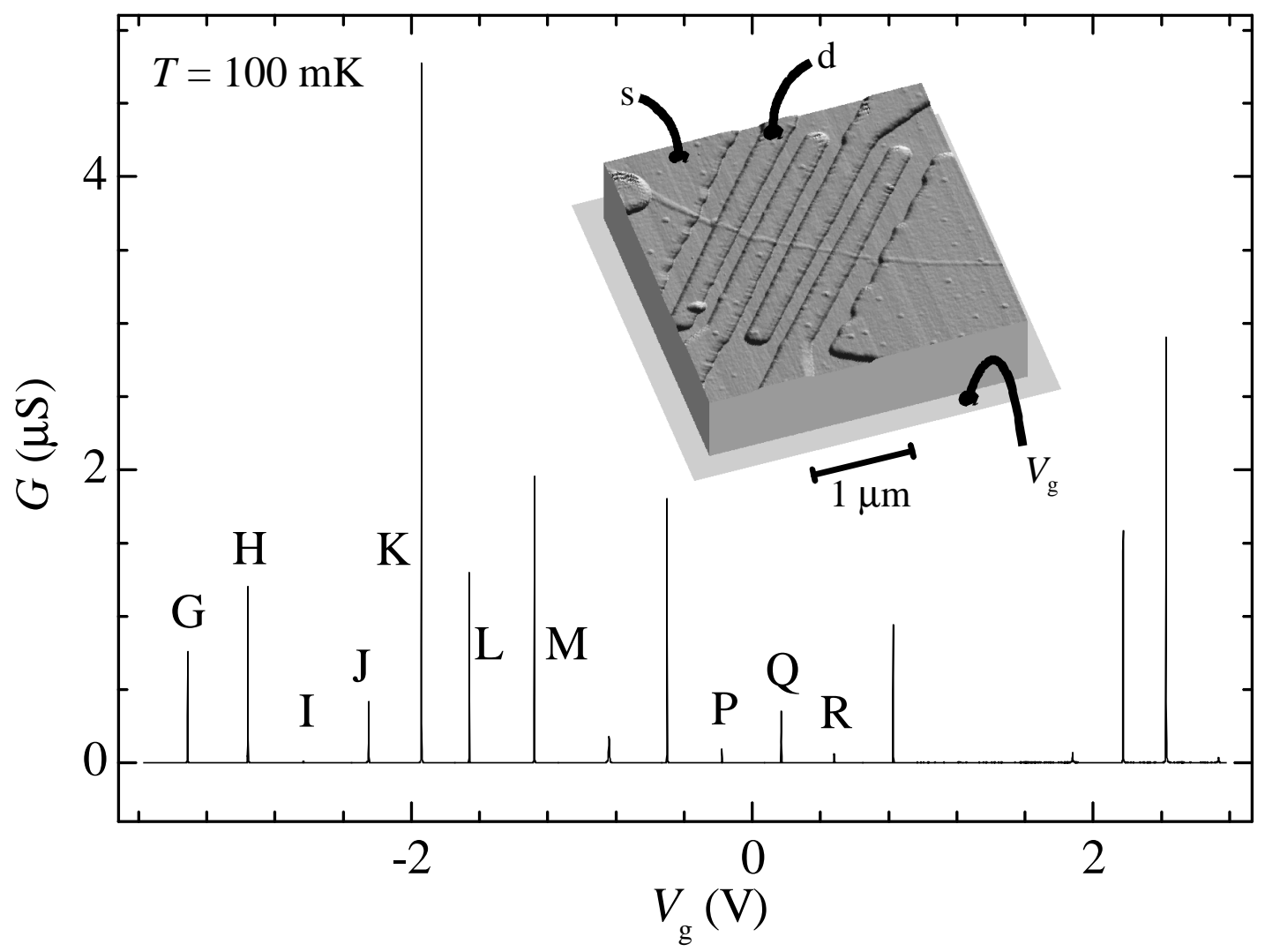

Figure 1

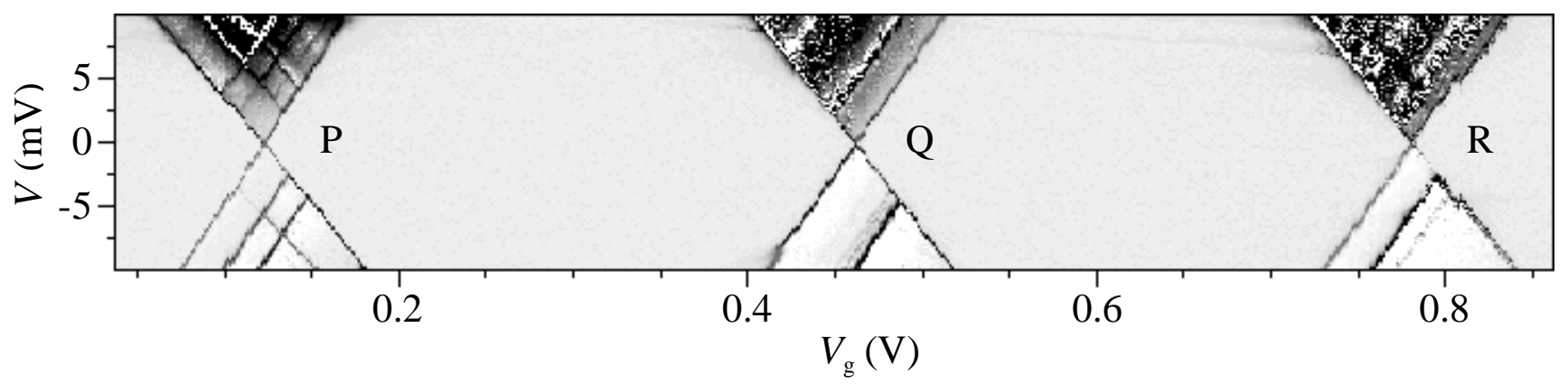

Figure 2 


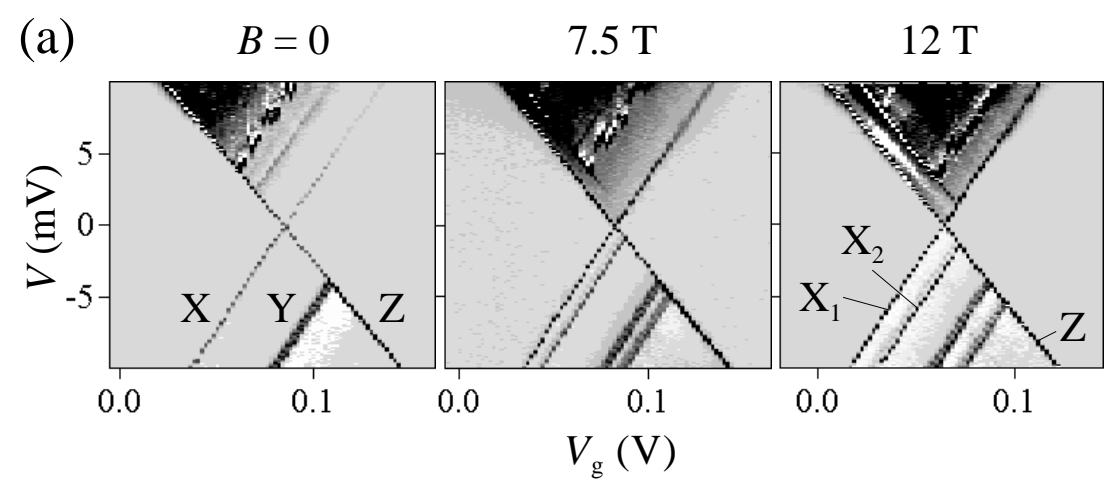

(b)
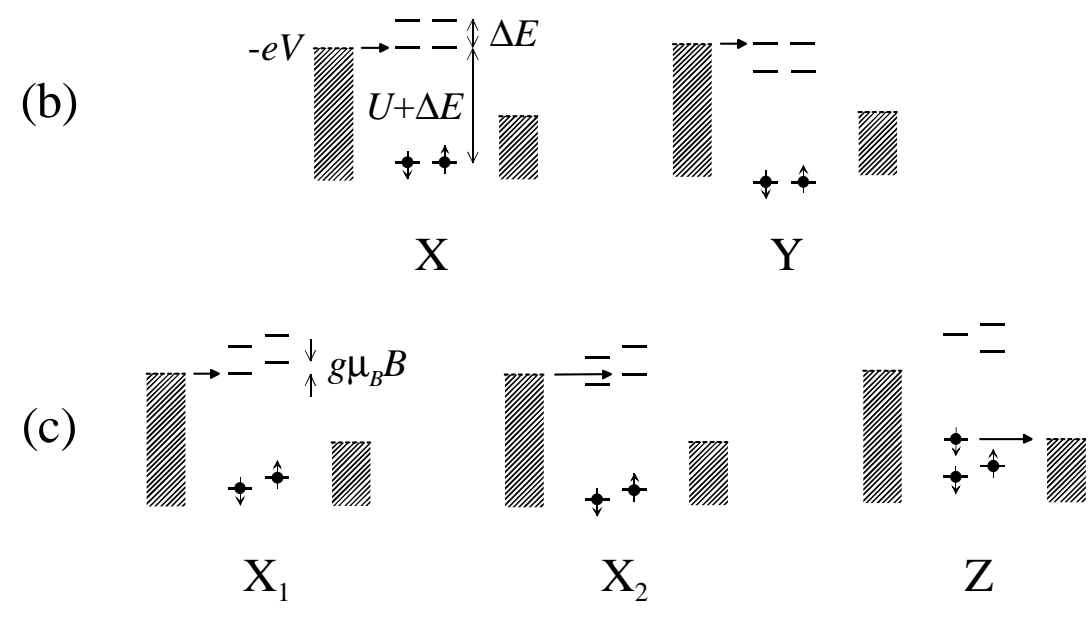

Figure 3

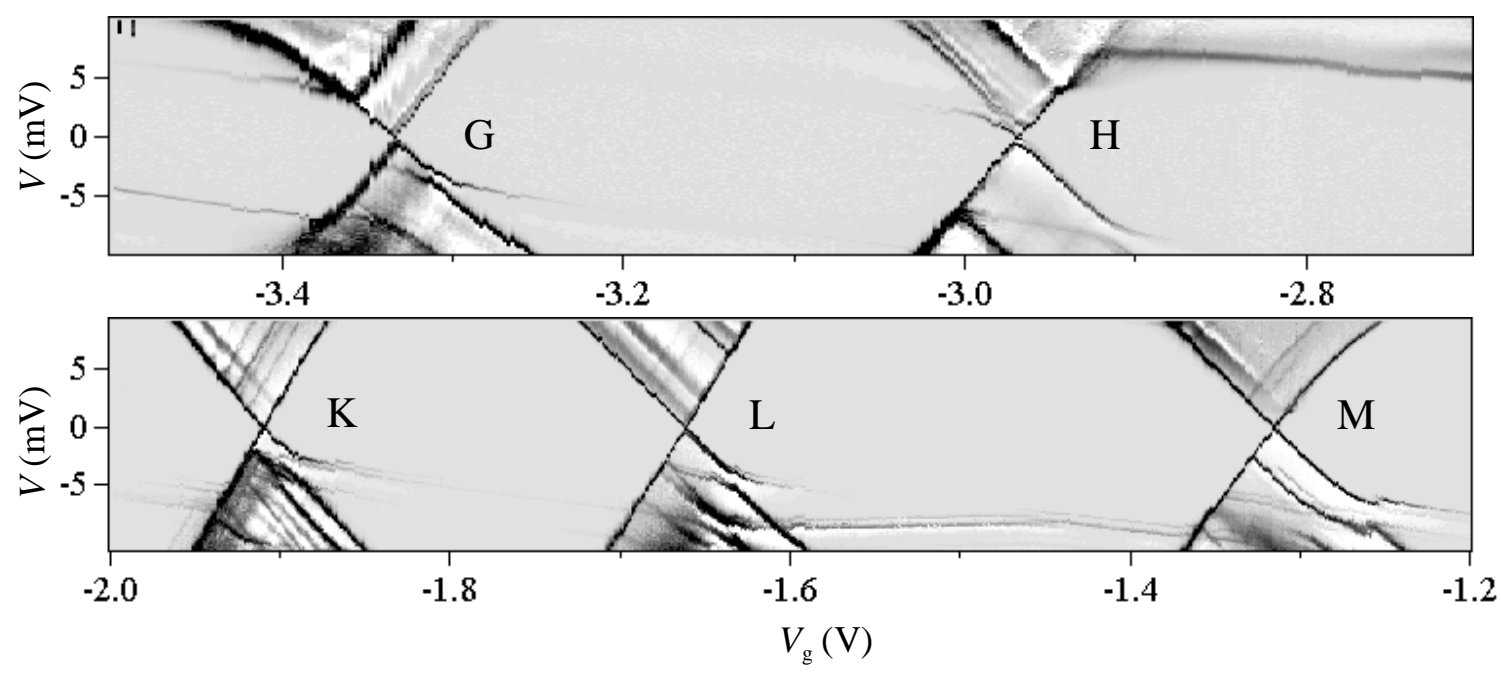

Figure 4 